\title{
Design of Sensors and Actuators Utilizing Computer Tools
}

\author{
Stefan J. Rupitsch ${ }^{1,3}$; Alexander Sutor ${ }^{1}$; Thorsten S. Albach ${ }^{1}$; Andreas Hauck ${ }^{1}$; Reinhard Lerch ${ }^{1}$; \\ Manfred Kaltenbacher ${ }^{1,2}$ \\ ${ }^{1}$ Chair of Sensor Technology, Friedrich-Alexander-University Erlangen-Nuremberg \\ Paul-Gordan-Str. 3-5, 91052 Erlangen, Germany \\ ${ }^{2}$ Applied Mechatronics, Institute of Smart Systems Technologies, Alpen-Adria University Klagenfurt \\ Universitaetsstr. 65-67, 9020 Klagenfurt, Austria \\ ${ }^{3}$ stefan.rupitsch@Ise.eei.uni-erlangen.de
}

\begin{abstract}
Computer tools are very useful for the design of sensors and actuators. Besides the design, these tools allow both, the characterization and optimization of devices without the need of extensive measurements as well as expensive fabrication of various prototypes. In this contribution, we present computer based design approaches of sensors and actuators which have been conducted at the Chair of Sensor Technology in recent years. As examples, a cavitation sensor made of ferroelectret material and a microacoustic loudspeaker are shown. The computer based design approaches are verified by measurement results for prototypes. Furthermore, we present the simulation based estimation of material parameters for piezoceramics commonly utilized for piezoelectric sensors and actuators.
\end{abstract}

\section{Introduction}

With the aid of computer tools, the effort for the design and optimization of sensors and actuators can be remarkably reduced. Moreover, these tools often allow the observation of effects inside the devices which are not accessible by measurement technology. Possible approaches for the computer design are based on the finite element method (FEM), boundary element method (BEM) as well as lumped circuit elements (LCE) [1, 2, 3]. In this contribution, we will concentrate on FEM and LCE. While for LCE the analogies between different physical fields (e.g., electric field, mechanic field) are applied to build up a network of lumped elements, FEM requires the coupling of these fields.

To predict the behavior of actuators and sensors, both, geometric dimensions of the devices and reliable material parameters of the involved materials are necessary [4]. Usually, sensors can be designed utilizing linearized material behavior. On the contrary, actuator devices require in the majority of cases the implementation of non-linear material laws for the computer based design. These material laws should allow the description of hysteresis behavior, drift and creep phenomena [5, 6].

A major task within the framework of the computer based design of sensors and actuators is the verification of the calculated results. In doing so, the reliability of the simulations for the design approach can be confirmed. An appropriate way to verify the simulation results is the comparison to measurements for prototypes of the devices. For instance, the electrical impedance, surface velocity and acoustic pressure can be utilized for those comparisons.

In this contribution, a brief introduction to the idea of LCE and FEM is given (Sec. 2). In Sec. 3 we present some examples for the computer based design of devices, which were conducted at the Chair of Sensor Technology in recent years. These examples are a MEMS-device (microacoustic loudspeaker) based on the magnetostrictive effect and a ferroelectret material utilized to emit and receive ultrasound waves. Finally, the simulation based identification (Inverse Method) of material parameters for piezoceramic materials is presented (Sec. 4). The paper concludes in Sec. 5 


\section{Approaches}

\section{Lumped Circuit Elements (LCE)}

The behavior of sensors and actuators can be described with the aid of lumped circuit elements (LCE), if the wavelength of the underlying physical fields (e.g., electrical field, mechanical field) are large compared to the dimensions of the devices [2, 3]. For instance, these lumped elements can be standard components of mechanics (spring, mass, damper) or electrical networks (resistor, inductor, capacitor). In order to convert the elements of the different physical fields, analogies (strictly speaking isomorphisms) are applied. Due to the fact that especially for electrical engineering several analysis tools (e.g., PSpice) are available on the market, these electrical lumped elements are commonly used. For the design of sensors and actuators, the coupling between the different physical fields requires so-called coupling systems (e.g., quadrupole) allowing the description of the interaction of the fields [2].

In contrast to other design approaches of sensors and actuators, LCE based modeling mostly leads to an elementary description of the device's behavior. Moreover, the optimization of the device design can be accomplished in a rather simple way. However, complex geometries of the device are accompanied by an extensive modeling effort.

\section{Finite Element (FE) Simulation}

Starting point of the finite element based design of sensors and actuators are the partial differential equations (PDE) describing the involved physical fields. Basically, the so-called strong formulation of the PDE is transformed by partial integration to the weak form [1]. Appropriate approximation methods of the physical quantities are then applied yielding an algebraic system of equations. Depending on the sought-after quantity, the solution of the equations is spatially and temporally discretized. Since sensors and actuators usually deal with different physical fields, reliable coupling mechanisms have to be considered within the framework of FEM. In many cases, the FE based description of this coupling mechanism leads to an iterative procedure $[1,3]$.

As against the LCE approach, the FEM can be applied even for complex geometries. Moreover, it is possible to handle open domain problems which occur for acoustic devices such as ultrasound transducers. For our FE simulations, we use CFS++ ("Coupled Field Simulation") which is a numerical simulation tool optimized for coupled field problems.

\section{Examples}

\subsection{Microacoustic Loudspeaker}

The microacoustic loudspeaker is based on magnetostriction which is a physical effect implying non-linearities and hysteresis behavior. In contrast to other actuation principles (e.g., electromagnetic, electrodynamic), magnetostriction provides both, high energy densities and contactless operation. Due to the low electrical impedance of magnetostrictive based actuators, only low driving voltages are required $[7,8]$.

The microacoustic loudspeaker is composed of two cantilever rows arranged opposite to each other. We fabricated structures with cantilever lengths and widths of $1250 \mu \mathrm{m}$ and $75 \mu \mathrm{m}$, respectively. One row consists of 58 cantilevers with a lateral spacing of $10 \mu \mathrm{m}$ between two neighboring cantilevers. Figure 1(a) depicts the cross section of a single cantilever which is mainly composed of a magnetostrictive layer (Vanadium Permendur $\mathrm{Fe}_{49} \mathrm{Co}_{49} \mathrm{~V}_{2}$ ) and an epoxy-based negative photoresist (SU8-3000) as passive layer [7, 8]. By applying an external magnetic field in $\mathrm{x}$-direction, the length of the magnetostrictive layer changes. Since the length of the passive layer remains unchanged, the magnetic field causes a bending of the cantilever consequently displacing air volume. If the magnetic field is temporally altered, a sound pressure can be generated primarily depending on the dimensions and amount of cantilevers.

Figure 1(b) shows the measurement setup utilized to investigate prototypes of the microacoustic loudspeaker. Basically, a motor driven wheel containing small permanent magnets is used for the generation of an external magnetic AC-field [7]. Thereby, the frequency of the field can be easily adjusted by the rotation speed of the wheel. The generated sound pressure is measured with a microphone (1/2", type4189, Bruel\&Kjaer) which is attached to a $2 \mathrm{ccm}$ standard measurement volume (RA0038, G.R.A.S, Denmark). 


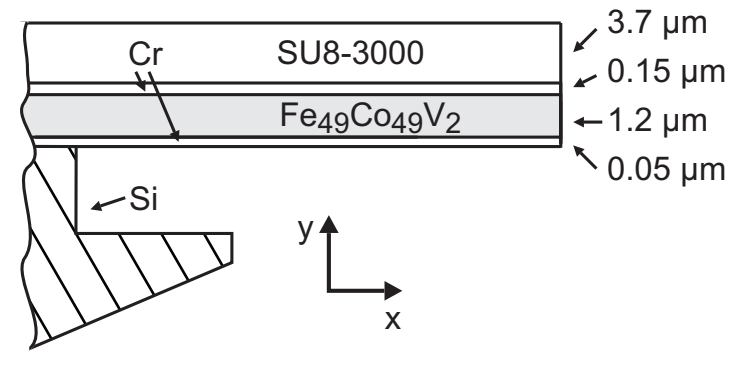

(a)

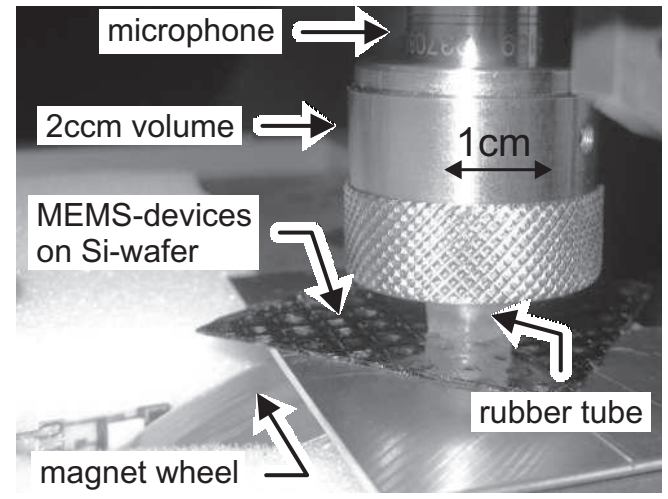

(b)

Figure 1: (a) Cross section of the cantilever mainly consisting of a magnetostrictive layer (Vanadium Permendur $\mathrm{Fe}_{49} \mathrm{Co}_{49} \mathrm{~V}_{2}$ ) and an expoxy-based negative photoresist (SU8-3000). Chromium (Cr) layers are used as adhesion promoter. (b) Measurement setup to investigate the microacoustic loudspeakers [7].

In Fig. 2(a) one can see measurement results for the frequency resolved sound pressure level (SPL) of the microacoustic loudspeaker. To avoid spurious reflection of acoustic waves, the measurements were conducted in an anechoic room.

Since the measurements and the fabrication of prototypes are rather extensive, there is a great demand for alternative approaches providing the frequency resolved sound pressure level of such devices. Hence, we developed a simulation model of the whole measurement assembly, which is based on the combination of lumped circuit elements and finite element simulation (see Fig. 2(b)) [2]. Due to the fact that the geometric dimensions of the device are small compared to the acoustic wavelengths in the audio range, the acoustic network can be described by lumped elements utilizing the electroacoustic analogy (acoustic pressure $p \Leftrightarrow$ electric voltage $U$; volume flux $q \Leftrightarrow$ electric current $I$ ). Moreover, the mechanical behavior of the moving cantilever representing an acoustic source is modeled by means of the electromechanical analogy (velocity $v \Leftrightarrow$ electric voltage $U$; force $F \Leftrightarrow$ electric current $I$ ) [2]. However, this description requires the mechanical impedance $Z_{\mathrm{M}}$ of the cantilever, too. That is why a finite element simulation was carried out yielding $Z_{\mathrm{M}}$ of the cantilever with respect to its excitation frequency. The coupling between the acoustical and mechanical network is performed by means of gyrator-type equations (Fig. 2(b)) [2, 7].

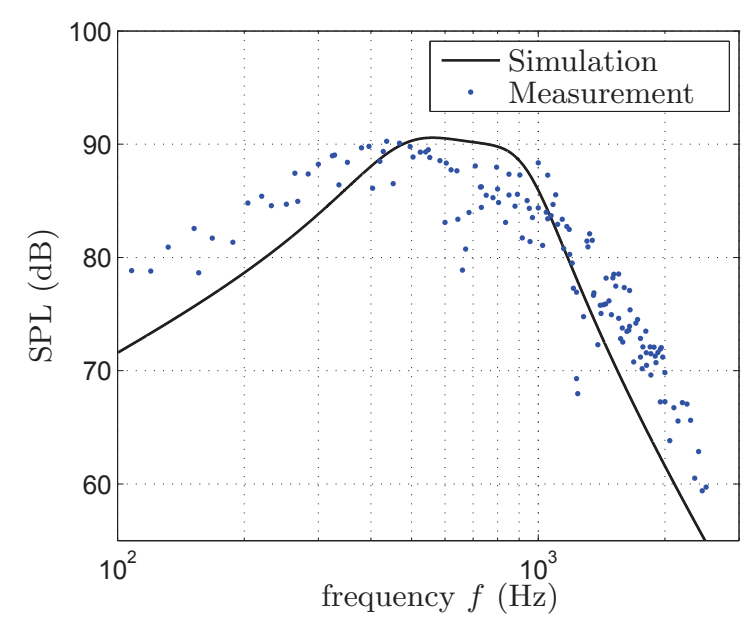

(a)

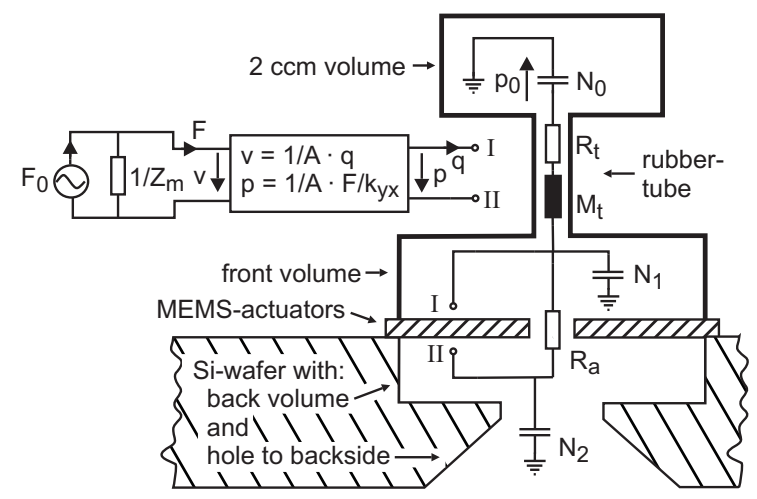

(b)

Figure 2: (a) Measurement and simulation results for the frequency resolved sound pressure level (SPL) of the microacoustic loudspeaker. (b) Simulation model of the measurement setup according to the lumped circuit element approach (mass element $M_{x}$, friction element $R_{x}$, acoustic compliance $N_{x}$, acoustic pressure $p_{x}$, volume flux $q$, velocity $v$, force $F$, mechanical impedance of the cantilever $Z_{\mathrm{M}}$ ) [7]. A scaling factor $k_{\mathrm{yx}}$ has to be applied, to map the appearing forces on the same physical direction.

As the results in Fig. 2(a) show, the simulation based approach yields reliable results for the frequency re- 
solved SPL. Thus, it is possible to predict the behavior of such devices without the need for both, extensive prototype fabrication and time consuming measurement process.

\subsection{Ultrasound Transducers based on Ferroelectret Material}

We utilized a ferroelectret material, namely the so-called EMFi (Electro-Mechanical-Film) material, to build up ultrasound transducers for different applications [3, 9]. Among other applications, it was used for an artificial bat head allowing the functional reproduction of the biosonar system found in bats and a sensor array for the investigation of cavitation effects in ultrasonic cleaning systems. Ferroelectret materials offer both, a high piezoelectric strain constant and a rather large bandwidth for sensor and actuator applications in water and air. Since the material exhibit a cellular structure (lenticular gas voids; see Fig. 3(a)), it is flexible and can therefore be used for curved transducers.

In order to obtain a FE simulation based description for the electrical and mechanical behavior of the ferroelectret material, we developed a microscopic as well as a macroscopic material model [9]. For instance, the microscopic model can be used to investigate the influence of the geometric dimensions of the lenticular gas voids on the piezoelectric strain constant and resonance frequency of the material in thickness direction. Due to the simulation effort, the microscopic model is not applicable for transducer structures with dimensions much larger than the lenticular voids. Against that, the macroscopic model allows the simulation based description of the global transducer behavior. However, for the macroscopic model, material parameter tensors (elastic stiffness, electric permittivity, piezoelectric coefficients) covering the global behavior are required. With a view to estimating these tensors, we applied the so-called Inverse Method (see Sec. 4) $[4,9]$.

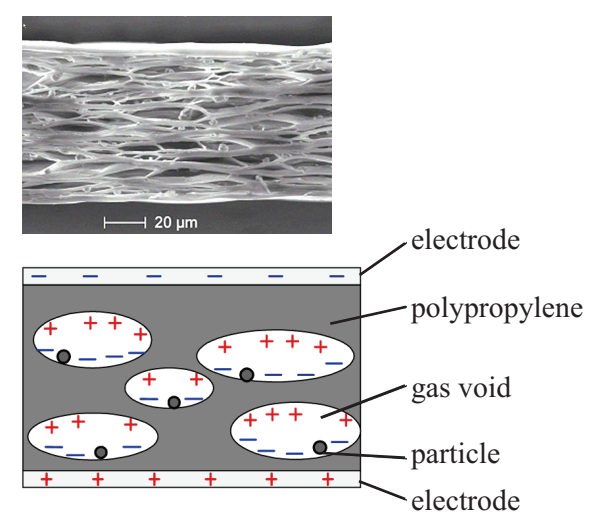

(a)

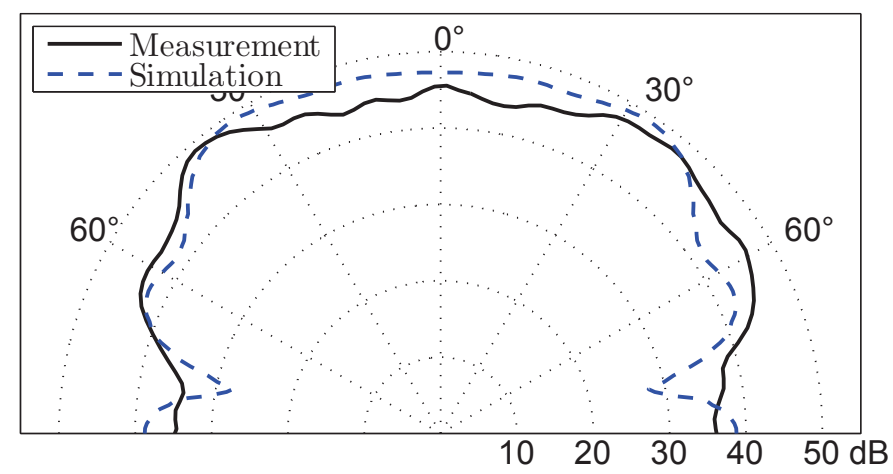

(b)

Figure 3: (a) Scanning electron micrograph of the cross section of an EMFi-material and schematic of the charge distribution inside a polarized film. (b) Measurements and simulations for the acoustic directivity pattern of an EMFi-foil which was glued on a cylinder (excitation voltage $U_{\mathrm{PP}}=800 \mathrm{~V}$; excitation frequency $f=100 \mathrm{kHz}$; distance $1 \mathrm{~m})$.

Figure 3(b) illustrates measurement and simulation results (macroscopic model) for the acoustic directivity pattern in air of an EMFi-material, which was glued on a cylinder. Note that for this simulation, a coupling of the electric, mechanic and acoustic field is indispensable. As the results show, the deviations between simulations and measurements are considerably small over a large angular range. Consequently, it is possible to replace the extensive measurements for other transducer geometries and alignments by means of numerical simulations.

As mentioned above, we utilized the EMFi-material to setup a sensor array which is used to investigate cavitation effects in ultrasonic cleaning systems [9, 10]. Figure 4(a) depicts the geometric dimensions of the array consisting of $4 \times 4$ elements and an image of the fabricated sensor which is protected against cavitation damage by a Parylene $C$ layer. Since for the developed sensor arrays both, the mechanical and electrical crosstalk are essential attributes, we conducted measurements and simulations (see Fig. 4(b)). According to the reciprocal behavior of sensing and actuation processes [2, 3], a single element was excited to determine the mechanical crosstalk within the sensor array. In the geometric range of the excited element, measurements and simulations coincide very well. The deviations outside of this element can mainly 
be ascribed to the electrical crosstalk between the signal lines and the sensor elements which was not considered in the simulation approach. However, a separate consideration of the mechanical and electrical crosstalk with the aid of measurements is hardly possible. Hence, the numerical simulation is the only way to quantify the different effects. By means of FE simulations, we were able to quantify both, the electrical crosstalk between the signal lines and between the sensor elements due to capacitive coupling $[9,10]$. For the case of underwater operation, the coupling capacity between the signal lines reaches values of $16 \mathrm{pF}$. On account of this fact, we utilized current amplifier circuits for the read-out of the sensor array.
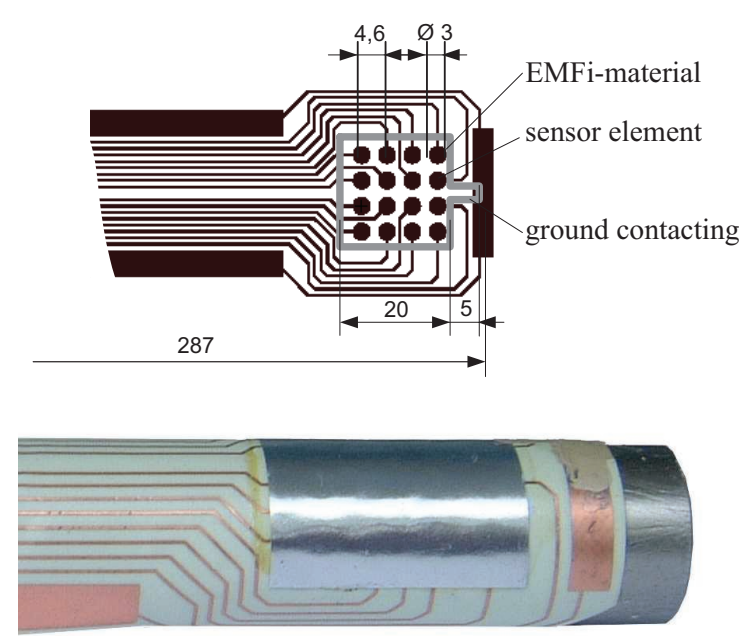

(a)

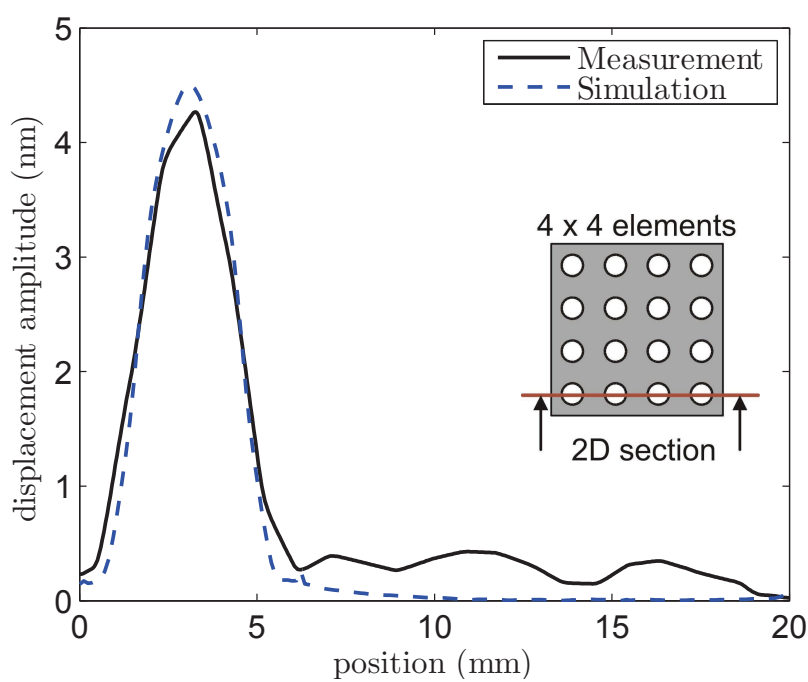

(b)

Figure 4: (a) Schematics of the geometric sensor structure and picture of the fabricated cavitation sensor (coated with Parylene C). (b) Measurements and simulations for the mechanical crosstalk within the sensor array along the profile line (excitation voltage $U_{\mathrm{PP}}=100 \mathrm{~V}$; excitation frequency $f=27.2 \mathrm{kHz}$ ).

\section{Simulation based Material Parameter Estimation for Piezoceramic Materials}

Besides the reliable modeling of physical fields which are involved in sensors and actuators, accurate material parameters are mandatory to simulate the behavior of the devices [1]. If precise simulation models as well as the required measurement equipment are available, numerical simulations can be applied to identify the properties of the device materials. In doing so, the material parameters are adjusted with the aid of a comparison of measurements and simulations [4].

Commonly, material parameters of piezoceramics (elastic stiffness constants, electric permittivity components, piezoelectric coefficients) are determined according to the IEEE-standard [4]. Thereby, the frequency resolved electrical impedance of samples exhibiting different shapes and geometries are analyzed. Within the framework of this standard, mono-modal vibrations of the samples are assumed which is strictly speaking an oversimplification. Since the manufacturers usually identify the material parameters of piezoceramics by means of the IEEE-standard, the provided parameters are oftentimes not appropriate for reliable simulations. In contrast to the usual method, we utilize a simulation based approach, namely the so-called Inverse Method, for material parameter estimation of piezoceramic materials [4]. Basically, the material parameters are adjusted in a convenient way with the aid of a comparison of measurements and simulation results. Both the frequency resolved electrical impedance and the frequency as well as spatial resolved surface normal velocity serve as input quantity for the method. While the impedance captures the global behavior of the piezoceramic device, the velocity provides local information.

Figure 5(a) depicts measurements and simulations of the frequency resolved electrical impedance for the radial mode of a piezoceramic disc (material Pz27; diameter $25.2 \mathrm{~mm}$; thickness $2.0 \mathrm{~mm}$ ). The results clearly show that the manufacturer's material parameters lead to considerable deviations between measurements and simulations. Against that, the parameters resulting from the Inverse Method yield reliable 
simulations. In Fig. 5(b) one can see a comparison of the spatially resolved surface normal velocity of the disc for the excitation frequency $1.0 \mathrm{MHz}$. Again, the Inverse Method provides material parameters which can be used for reliable calculations of the disc's behavior. However, the parameters from the manufacturer are not suitable to describe the mechanical behavior of the piezoceramic device.

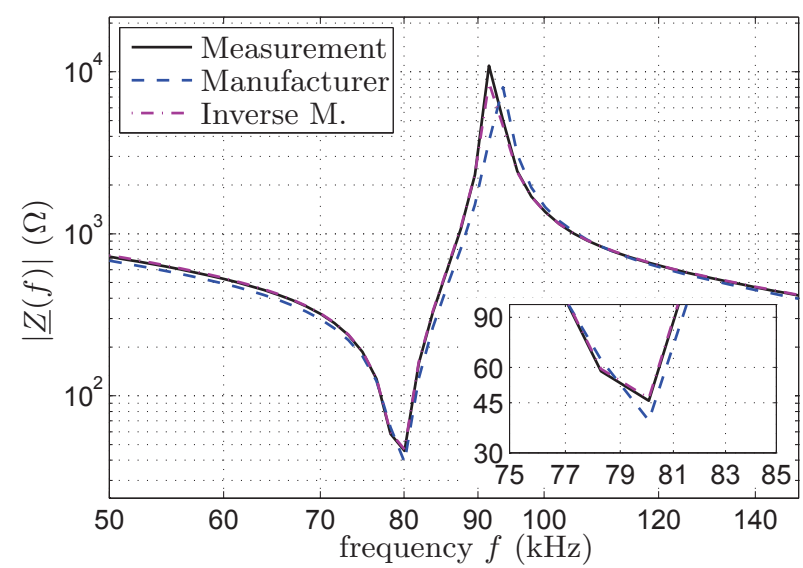

(a)

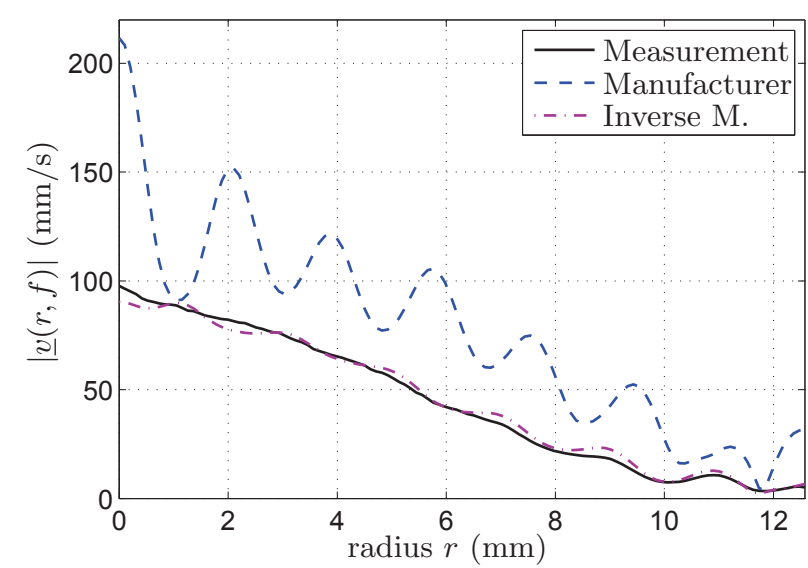

(b)

Figure 5: (a) Measurements (acquired with impedance analyzer Hewlett Packard HP4194A) and simulations for the frequency resolved electrical impedance for the radial resonance of a piezoceramic disc (material Pz27; diameter $25.2 \mathrm{~mm}$; thickness $2.0 \mathrm{~mm}$ ). (b) Measurements (acquired with laser-scanning-vibrometer Polytec PSV300) and simulations for the spatially resolved surface normal velocity of the disc (excitation voltage $U_{\mathrm{PP}}=10 \mathrm{~V}$; excitation frequency $f=1.0 \mathrm{MHz}$ ) [4].

\section{Conclusion}

We presented design approaches for sensors and actuators utilizing computer tools. The main benefit of these approaches lies in the reduction of both, measurement efforts and prototype manufacturing. In this contribution, the computer based design was shown for a microacoustic loudspeaker and a ferroelectret material utilized for ultrasound transducers. Therefore, different design approaches were chosen, namely lumped circuit elements and the finite element method. Comparisons of measurement and simulation results clearly verify the computer based design methods. Finally, the idea of the simulation based material parameter identification for piezoceramics was introduced.

\section{References}

[1] M. Kaltenbacher, Numerical Simulation of Mechatronic Sensors and Actuators, Springer Berlin Heidelberg, 2007.

[2] R.G. Ballas, G. Pfeifer, and R. Werthschützky, Elektromechanische Systeme in Mikrotechnik und Mechatronik, Springer Berlin Heidelberg, 2009.

[3] R. Lerch, G. Sessler, and D. Wolf, Technische Akustik, Springer Berlin Heidelberg, 2009.

[4] S.J. Rupitsch, R. Lerch, "Inverse Method to estimate material parameters for piezoceramic disc actuators," Applied Physics A: Material Science \& Processing, vol. 97, pp. 735-740, 2009.

[5] A. Sutor, S.J. Rupitsch, and R. Lerch, "A Preisach based hysteresis model for magnetic and ferroelectric hysteresis of piezoelectric materials," Applied Physics A: Material Science \& Processing, vol. 100, pp. 425-430, 2010.

[6] F. Wolf, A. Sutor, S.J. Rupitsch, and R. Lerch, "Modeling and measurement of creep- and rate-dependent hysteresis in ferroelectric actuators," Sensors and Actuators A: Physical, in press, 2011.

[7] T. Albach, P. Horn, A. Sutor, and R. Lerch, "Sound generation using a magnetostrictive micro actuator," Journal of Applied Physics, in press, 2011.

[8] T. Albach, A. Sutor, and R. Lerch, "Untersuchung an magnetostriktiven Mikroaktoren," Technisches Messen, vol. 77, pp. 67-73, 2010.

[9] S.J. Rupitsch, R. Lerch, J. Strobel, and A. Streicher, "Ultrasound transducers based on ferroelectret materials," IEEE Transactions on Dielectrics and Electrical Insulation, vol. 18, pp. 69-80, 2011.

[10] J. Strobel, S.J. Rupitsch, and R. Lerch, "Ferroelektretsensor zur Messung der Kavitation in Ultraschallreiningungsbädern," Technisches Messen, vol. 76, pp. 487-495, 2009. 